\title{
Study of RF Signal Attenuation of Human Heart
}

\author{
Kedar Nath Sahu, ${ }^{1}$ Challa Dhanunjaya Naidu, ${ }^{2}$ M. Satyam, ${ }^{3}$ and K. Jaya Sankar ${ }^{3}$ \\ ${ }^{1}$ Department of Electronics and Communication Engineering, Stanley College of Engineering and Technology for Women, \\ Hyderabad, Telangana 500 001, India \\ ${ }^{2}$ Department of Electronics and Communication Engineering, VNR Vignana Jyothi Institute of Engineering and Technology, \\ Hyderabad, Telangana 500 090, India \\ ${ }^{3}$ Department of Electronics and Communication Engineering, Vasavi College of Engineering, Hyderabad, Telangana 500 031, India \\ Correspondence should be addressed to Kedar Nath Sahu; knsahu72@gmail.com
}

Received 28 July 2014; Revised 15 December 2014; Accepted 9 February 2015

Academic Editor: Kamran Iqbal

Copyright (C) 2015 Kedar Nath Sahu et al. This is an open access article distributed under the Creative Commons Attribution License, which permits unrestricted use, distribution, and reproduction in any medium, provided the original work is properly cited.

A study of ultrawideband pulse propagation modeling through human body for all frequencies from 0.1 to $10.5 \mathrm{GHz}$ is presented. Reflection coefficient and signal attenuation are computed from the model considering the variation of heart dimension with respect to time unlike a fixed dimension of heart used in earlier models. The performance of cardiac activity is studied from the change of signal attenuation. This estimation may help in the design of a noninvasive diagnostic system using ultrawideband of frequencies.

\section{Introduction}

Human heartbeat performance is the basis for many modern potential applications because it is impossible to suppress the heartbeat related motion. Some of the major applications include monitoring of heartbeat of a newborn infant in a pediatric clinic, burnt victims, and remote monitoring of the health condition of a patient as well as the old people who are asleep. As the heart involves a very small amplitude motion, its detection becomes more challenging. Any contact based method, for example, an electrocardiogram (ECG), requires a physical contact with the body of the human subject and hence is invasive. However, an invasive method suffers from the drawbacks such as being difficult to apply in case of human infant subjects who are very susceptible to sudden infant death syndrome (SIDS), intolerance of placing electrodes on the body of burnt victims, problem of countermeasures, and the effect on the autonomic nervous system (ANS) during lie detection. Detection and/or monitoring of cardiac performance using ultrawideband (UWB) radar technique is becoming of great importance as it is noninvasive and remote. Furthermore, UWB radars have several special features such as environmental friendliness, very low electromagnetic energy emission, high miniaturization capability, very low cost, and high resolution. UWB radar transmits a sequence of very short pulses over a large bandwidth unlike the continuous-wave (CW) radars which transmit a continuouswave signal at a particular frequency. The radio frequency (RF) signal returned to the radar receiver after being reflected from the human subject contains the information about the functional pattern of the human heart. Therefore, the study of UWB wave propagation aspects into a human thorax model helps to obtain the state of the human heart. The model predicted estimation will be useful to estimate how much power is needed for a specific purpose and also to get an indication as to whether the person is living or dead. The accuracy of a study of this kind depends on an appropriate and effective propagation model.

UWB propagation into the layered models developed earlier $[1,2]$ was identified for not considering the effect of multiple reflections. In our previous work [3], propagation of UWB pulses in the human tissues was studied for a fixed radar system placed on the room wall at a distance of one meter from the subject to be monitored. A multilayer planar model of human thorax was used to predict UWB signal attenuation considering signal propagation on the air-skinheart-air path. The multilayered model used the frequency dependent dielectric properties of layered tissue mediums 


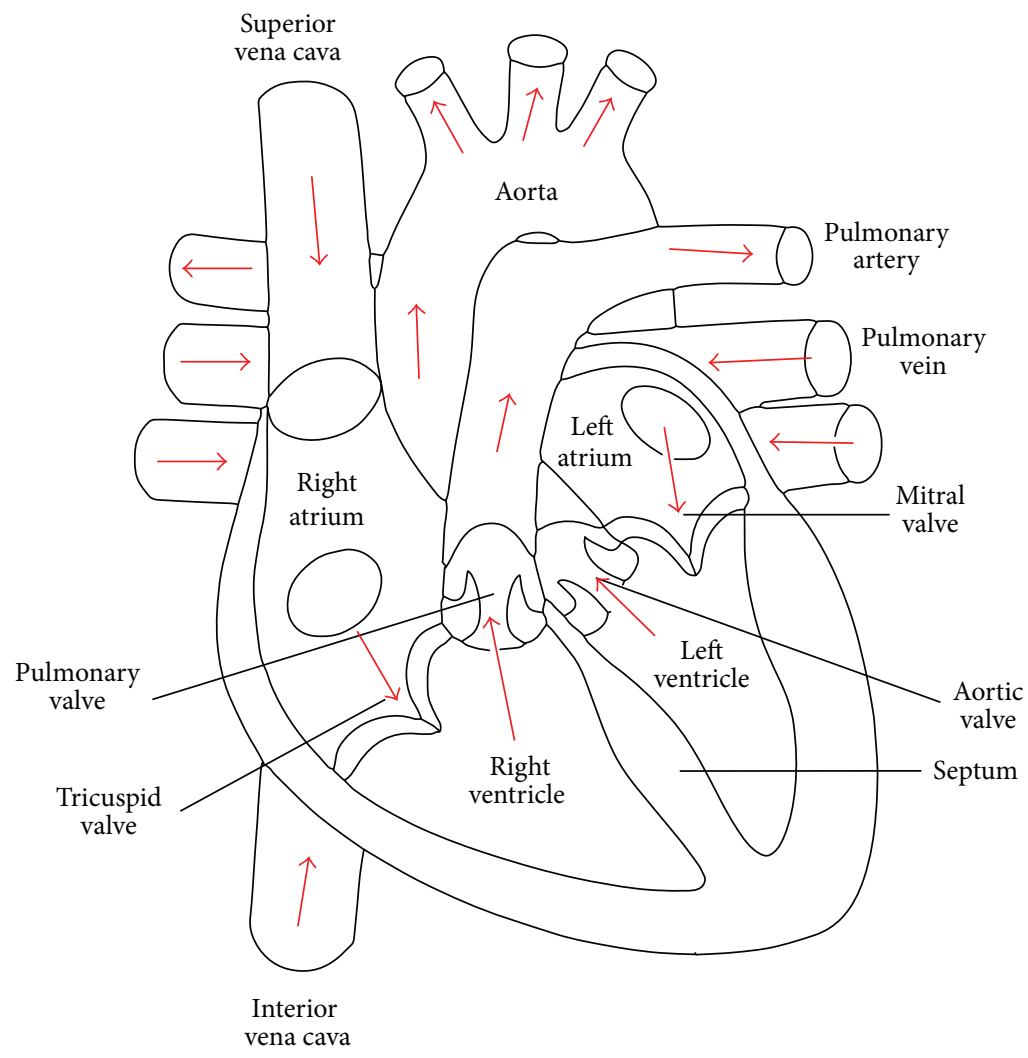

FIgURE 1: Transverse section of human heart [5].

over an ultrawideband similar to the frequency dependent model developed by [2] but was distinct due to the fact that the issue of multiple reflections ignored by the earlier models $[1,2]$ was accounted for by developing the impedance transformation model. This earlier idea was to develop a reasonably simple radar and the signal attenuation analysis was done up to the heart tissue in the thorax model. But electromagnetic energy leaks to the tissues through and beyond the heart up to the posterior skin as well. Therefore, the tissues beyond heart also contribute further to the signal attenuation which needs to be studied. A similar kind of model that considered tissues up to the posterior skin as well as the inclusion of the effect of multiple reflections was found in the literature [4].

In this paper, we have extended the analysis by considering a more standard anatomical full body model to develop a multilayer planar model and the dispersive behavior of electromagnetic properties of human tissues. In order to accommodate the frequencies of the Federal Commission for Communication (FCC) approved UWB (3.1 to $10.6 \mathrm{GHz}$ ) the signal attenuation is evaluated for typical frequencies from 0.1 to $10.5 \mathrm{GHz}$. In order to validate the considered model, the signal attenuation as a function of frequency will be evaluated and compared with the results obtained in $[2,4]$. Due to the particular applications of UWB radar in medicine, it is fundamental to investigate the propagation of UWB pulses in human tissues with reference to both the safety issues and the determination of optimum signal to monitor the cardiopulmonary activity.

\section{The Planar Thorax Model}

In order to evaluate the propagation characteristics for varied heart dimensions the time dependence of heart motion was studied and described in the following section with a brief description about the constitution of heart.

2.1. The Human Heart Structure. This is a hollow, cone shaped, four-chambered (left and right atria, left and right ventricles) muscle located between the lungs and behind the sternum. Two-thirds of the heart is located to the left of the midline of the body and the remaining one-third is placed to the right. According to the medical illustrations, right and left correspond to the person's right and left assuming that the person is looking at us.

The transverse cross-sectional view of heart structure [5] as shown in Figure 1 consists of left ventricle wall, left ventricle cavity, interventricular septum, right ventricle cavity, and right ventricle wall. The left ventricle (LV) and the right ventricle (RV) are the longer cavities in the human heart. The left ventricle has a thicker wall than the right ventricle.

2.1.1. The Cardiac Cycle. The heart function alternates between contraction and relaxation in a concerted pattern called the cardiac cycle. One cardiac cycle consists of one complete diastole and one complete systole. Diastole is the phase of the cardiac cycle during which the chambers of the heart relax and the ventricles dilate allowing the blood to flow in. Systole is the phase of the cardiac cycle during which the 
ventricles contract pumping the blood into the aorta and the pulmonary artery. At the start of the diastole, the heart muscle is relaxed and blood flows into the atria. At the end of diastole, both atria contract simultaneously and this helps to fill the ventricles with blood immediately prior to the systole. One cardiac cycle is completed in 0.8 seconds (i.e., in less than one second). A systole is one complete contraction phase of the cardiac cycle and a diastole is one complete relaxation phase.

2.1.2. The Cardiac Dimension and Its Variation. The heart and its performance are commonly measured in terms of onedimensional distances. The left ventricle end diastole (LVED) is the length measured at the end of diastole (i.e., when the heart is fully relaxed) and normally corresponds to the largest cardiac dimension. Similarly, the left ventricle end systole (LVES) is the length measured at the end of systole (i.e., when the heart is fully contracted) and corresponds to the smallest cardiac dimension. The ventricular cavity, interventricular septum, ventricular-free wall thickness, and their changes with respect to time during the cardiac cycle have been measured using various methods such as echocardiography, angiography, and cine MRI, as adopted in [6-12]. The instantaneous time of the dimensions and the time-rates of change in the wall thickness, cavity area, and transverse dimension during isovolumic relaxation (end-diastole) and contraction (end-systole) for normal subjects are estimated and reported in [6]. Results of the normal diameters of the cardiac cavities and the ventricular, interventricular wall thickness values as reported in literature are mentioned as follows.

The end-diastolic LV cavity wall thickness is $0.9 \pm 0.2 \mathrm{~cm}$ which is almost close to the value $0.8 \pm 0.2 \mathrm{~cm}$ as reported in [9] increasing to $2.0 \pm 0.5 \mathrm{~cm}$ at the end of systole [6]. The LVED cavity diameter $4.9 \pm 0.4 \mathrm{~cm}$ [7] is close to $5.16 \pm$ $0.46 \mathrm{~cm} \mathrm{[8]} \mathrm{and} 50 \mathrm{~mm}$ [9] and consistent in the normal range $3.3-5 \mathrm{~cm}$ of [7]. At the end of systole, the LV cavity gets a reduction of $2.2 \pm 0.4 \mathrm{~cm} \mathrm{[6]} \mathrm{and} \mathrm{thus} \mathrm{the} \mathrm{cavity}$ diameter becomes about $2.7 \mathrm{~cm}$. This value is as close as $3.38 \pm$ $0.36 \mathrm{~cm}$ as mentioned in [7], $4 \mathrm{~cm}$ as in [8], and in the normal range $2.78-5.4 \mathrm{~cm}$ as reported in [11]. The thickness value of interventricular septum is $8.3 \mathrm{~mm}$ which satisfies the normal range of $7-11 \mathrm{~mm}$ and is in consistency with $10.3 \pm 0.5 \mathrm{~mm}$ as reported in [10]. The RV cavity diameter at the end of diastole is $37.1 \pm 5.9 \mathrm{~mm}$, that is, $3.7 \pm 0.59 \mathrm{~cm}$, and is equal to $2.8 \mathrm{~cm}$ at the end of systole as mentioned in [7] and its adjacent wall (the posterior wall) thickness at the end of diastole is $0.8 \pm 0.2 \mathrm{~cm}$ as found in [9] which is close to the value $10.2 \pm 0.5 \mathrm{~mm}$ as reported in [10] and correspondingly at the end of systole, this value is $1.3 \pm 0.2 \mathrm{~cm}$ as given in [9]. So far as the rate of change of dimension $(d D / d t)$ is concerned, the peak systolic $d D / d t$ is $13 \pm 5 \mathrm{~cm} / \mathrm{s}$ and the peak diastolic $d D / d t$ is $16 \pm 4 \mathrm{~cm} / \mathrm{s}$ as mentioned in [6]. The total thickness of heart at the end of diastole, at the end of systole, and during the interval from the end of diastole to the end of systole is the sum of the instantaneous thickness values of left ventricle wall (anterior wall), left ventricle (LV) cavity, septum thickness, right ventricle (RV) cavity, and the right ventricle wall (posterior wall). Based on the changes of cavity dimension, wall thickness with time as well as their peak rates of change as reported in [6], the total heart size (in millimeter)

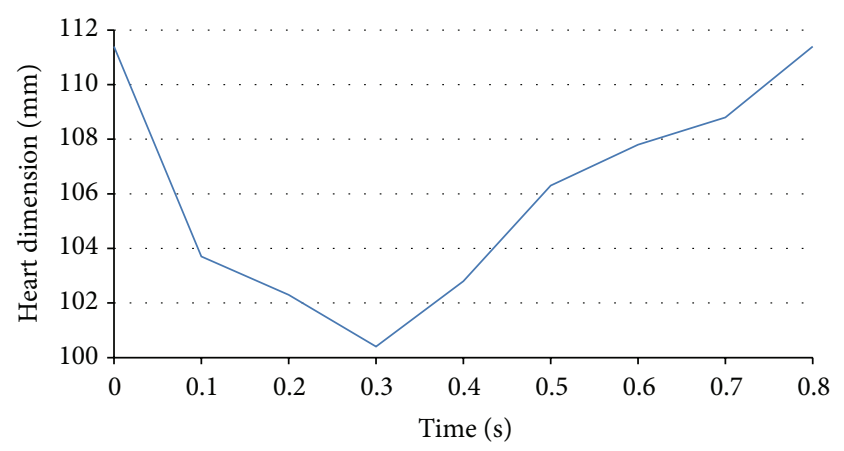

FIGURE 2: Variation of transverse dimension of normal human heart with time during a cardiac cycle.

TABLE 1: Instantaneous dimensions of human heart.

\begin{tabular}{lcc}
\hline Time $(\mathrm{s})$ & Heart size $(\mathrm{mm})$ & Heart activity \\
\hline 0 & 111.4 & End of diastole and start of systole \\
\hline 0.1 & 103.7 & Systole \\
0.2 & 102.3 & \\
\hline 0.3 & 100.4 & End of systole and start of diastole \\
\hline 0.4 & 102.8 & Diastole \\
0.5 & 106.3 & \\
0.6 & 107.8 & \\
0.7 & 108.8 & End of diastole \\
\hline 0.8 & 111.4 & \\
\hline
\end{tabular}

during systole and diastole is obtained for one complete cardiac cycle of $0.8 \mathrm{~s}$ as shown in Table 1 and depicted in Figure 2.

2.2. Multilayered Human Thorax Model. The model discussed in [3] included a few major tissues from chest skin up to heart only, for the study of signal attenuation. In this work, we extended the analysis by including the significant tissues beyond the heart and right up to the posterior skin as shown in Figure 3.

The same Visible Human Project based anatomical thickness values of the tissue layers as used in $[1,3]$ are considered for this model. The thickness of dry skin of $1.5 \mathrm{~mm}$, average infiltrated fat of $9.6 \mathrm{~mm}$, muscle of $13.5 \mathrm{~mm}$, cartilage of $11.6 \mathrm{~mm}$, deflated lung of $5.78 \mathrm{~mm}$, and the different heart thickness values corresponding to the different instants of time of the cardiac cycle (Table 1) have been considered. The same tissues of these thickness values are considered behind the heart as well. The dispersive behavior of human tissues has been taken into account through the Cole-Cole model using the parameters computed in Gabriel's data book of dielectric properties of tissues [13] and also reported in [14]. The variations of the dispersive dielectric properties as a function of frequency at all frequencies from 0.1 to $10.5 \mathrm{GHz}$ are plotted as shown in Figure 4. It is seen that, over the entire band of frequencies considered, as the frequency increases, the relative permittivity decreases (Figure 4(a)) but the conductivity increases (Figure 4(b)). This implies that the other propagation parameters such as attenuation constant, 


\begin{tabular}{|c|c|c|c|c|c|c|c|c|c|c|c|}
\hline & & & & 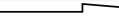 & 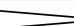 & & & & & & \\
\hline Air & Skin & Fat & Muscle & Cartilage & Lung & Heart & Cartilage & Muscle & Fat & Skin & Air \\
\hline 1000 & 1.5 & 9.6 & 13.5 & 11.6 & 5.78 & & 11.6 & 13.5 & 9.6 & 1.5 & \\
\hline
\end{tabular}

(a)

\begin{tabular}{|c|c|c|c|c|c|c|c|c|c|c|c|}
\hline \multicolumn{12}{|c|}{ 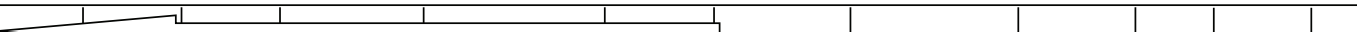 } \\
\hline Air & Skin & Fat & Muscle & Cartilage & Lung & Heart & Cartilage & Muscle & Fat & Skin & Air \\
\hline 1000 & 1.5 & 9.6 & 13.5 & 11.6 & 5.78 & & 11.6 & 13.5 & 9.6 & 1.5 & \\
\hline
\end{tabular}

(b)

FIGURE 3: Tissue structure for EM modeling of human body: (a) forward propagation; (b) backward propagation. Figures in the diagram indicate the thickness of the tissue layers in millimeter.

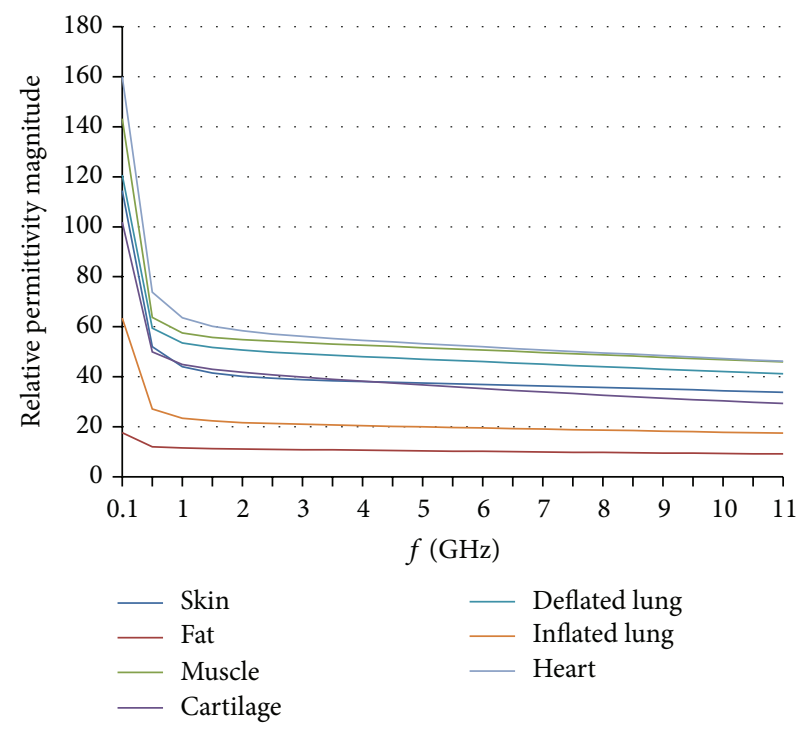

(a)

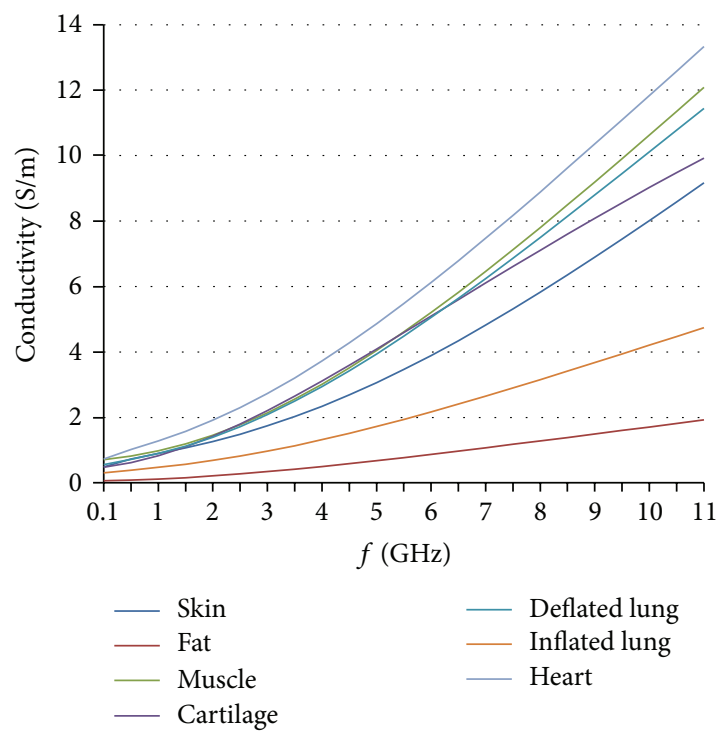

(b)

FIGURE 4: Variation of (a) relative permittivity; (b) conductivity of tissues with frequency.

phase-shift constant, magnitude of intrinsic impedance, and wave velocity also increase with frequency.

\section{Analysis of UWB Pulse Propagation into the Model}

As biological tissues are lossy media from an electromagnetic point of view, they are characterized in terms of the propagation parameters discussed in Section 2.2. These characteristic parameters are highly frequency dependent. The incident, reflected, and transmitted powers at any interface of the multilayered thorax model (Figure 3) are the result of a net forward wave due to the multiple reflections taking place in the previous interfaces and the reflected wave may be considered as the effect of all the multiple reflections occurring at the boundary. Therefore, the net incident, reflected, and transmitted powers at all tissue interfaces are calculated at typical frequencies of the ultrawideband range from 0.1 to $10.5 \mathrm{GHz}$ using the following power relations given by (1) through (3) as described in [15]:

Net incident power, $P_{i}^{+}=\frac{\left(E_{1}^{+}\right)^{2}}{2 \eta_{1}}$

Net reflected power, $P_{r}^{-}=\frac{\left(\Gamma E_{1}^{+}\right)^{2}}{2 \eta_{1}}=|\Gamma|^{2} \frac{\left(E_{1}^{+}\right)^{2}}{2 \eta_{1}}=|\Gamma|^{2} P_{i}^{+}$,

Net transmitted power, $P_{t}^{+}=\left(1-|\Gamma|^{2}\right) P_{i}^{+}$,

where $|\Gamma|$ is the reflection coefficient magnitude at an interface.

In the event of the wave reflection from such multiple interfaces, the impedance transformation method considers the complicated sequence of multiple reflections in every layer as explained in [15] assuming the normal incidence 


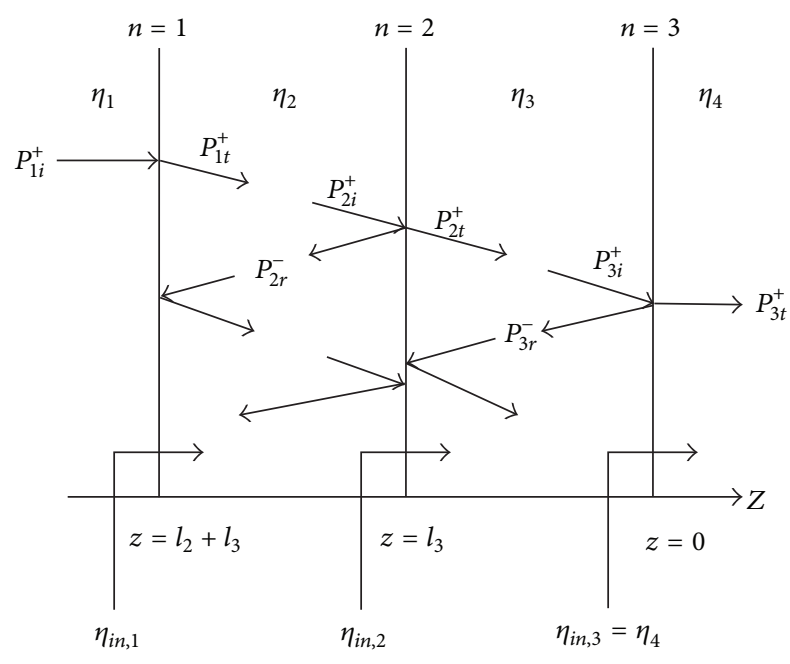

FIGURE 5: Planar impedance transformation model for a three-interface, four-layer case.

of a plane wave on every interface. A three-interface, fourlayer configuration of the planar impedance transformation model is as shown in Figure 5. Using the boundary conditions on either side of an interface, the effective input impedance offered by all subsequent layers to the right of every interface can be computed as obtained in [15]. The effective input impedance at the interfaces $n=3,2$ and 1 can be given by (4) through (6), respectively. Using the input impedance values calculated in this way, the reflection coefficient can then be calculated at every interface; for example, reflection coefficient at interface 1 can be as given by (7).

At interface 3, the effective input impedance is

$$
\eta_{\text {in, } 3}=\eta_{4}
$$

At interface 2, the effective input impedance is

$$
\eta_{\mathrm{in}, 2}=\eta_{3} \frac{\eta_{4} \cos \beta_{3} l_{3}+j \eta_{3} \sin \beta_{3} l_{3}}{\eta_{3} \cos \beta_{3} l_{3}+j \eta_{4} \sin \beta_{3} l_{3}}
$$

and at interface 1 , the effective input impedance becomes

$$
\eta_{\mathrm{in}, 1}=\eta_{2} \frac{\eta_{\mathrm{in}, 2} \cos \beta_{2} l_{2}+j \eta_{2} \sin \beta_{2} l_{2}}{\eta_{2} \cos \beta_{2} l_{2}+j \eta_{\mathrm{in}, 2} \sin \beta_{2} l_{2}}
$$

Then, reflection coefficient at interface 1 can be expressed as

$$
\Gamma_{1}=\frac{\eta_{\mathrm{in}, 1}-\eta_{1}}{\eta_{\mathrm{in}, 1}+\eta_{1}}
$$

where $\eta_{1}, \eta_{2}, \eta_{3}$, and $\eta_{4}$ are the intrinsic impedances; $\beta_{2}$ and $\beta_{3}$ are the wave numbers of the respective layers; $l_{2}$ and $l_{3}$ are the thickness values of layers 2 and 3 , respectively.

Similarly, considering the eleven-layered tissue system model (Figure 3), the input impedance and the reflection coefficient corresponding to every tissue interface are obtained using a MATLAB program.

Thus, in order to study the behavior of the backscattered field from a human body illuminated by the plane electromagnetic waves from a radar transmitter, we simplified

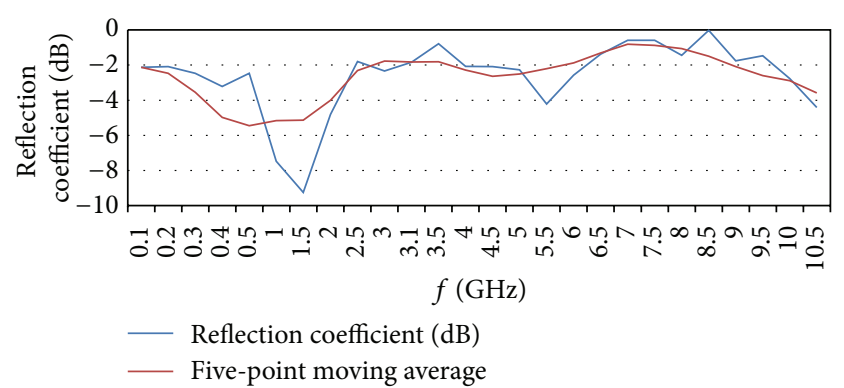

Figure 6: Reflection coefficient from heart at the end of diastole. A five-point moving average is superimposed for a clearer understanding.

the problem by modeling the human body as a series of biological tissue layers of complex permittivity. Knowing the permittivity of the tissue materials and by utilizing the basic principles of electromagnetic wave propagation in accordance with the physical processes, the power received by the radar receiver, the reflection coefficients at every interface, and signal power attenuation of the heart are determined.

\section{Results and Discussion}

When the power carried by the radar wave is incident on any interface, " $n$," separating the two tissue mediums " $n$ " and " $n+1$," part of it is transmitted to the next layer in the same forward direction, known as the transmitted power, and the remaining power is reflected into its previous layer in the backward direction known as the reflected power component. The amount of power reflected from every interface keeps getting retransmitted in a backward propagation mode and is finally received at the radar receiver. Such retransmitted power components from each of the interfaces received by the radar receiver in a backward propagation mode are called the backward reflected power. The transmitted power or the reflected power, respectively, through or from every interface during either mode of propagation, forward or backward, is multiplied by the power attenuation factor of the corresponding layer before entering into the next tissue layer.

(i) Reflection Coefficient. The characteristic behavior of the incident and reflected signals at every tissue interface of the planar eleven-layer model (Figure 3) based on the impedance transformation approach as a function of frequency is computed using MATLAB. Considering the completely relaxed state of the heart at the end of diastole, the variation of reflection coefficient of heart wall with frequency is plotted as shown in Figure 6. A five-point moving average is superimposed for clear understanding.

It is observed that the average reflection coefficient is as close as $-3 \mathrm{~dB}$ (approximately) over the whole band of frequency. This means that about half of the electromagnetic power incident on the model is reflected back and the other half is transmitted into the body. Moreover, the reflection coefficient value is negative at any frequency because the impedance offered by the human body is less than 


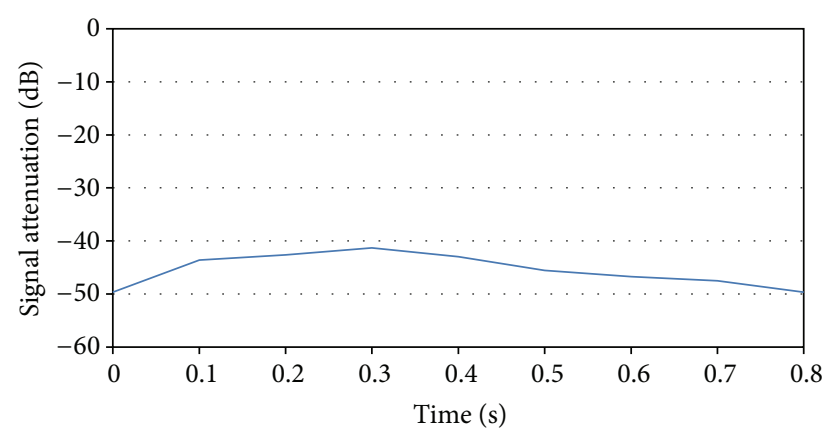

(a)

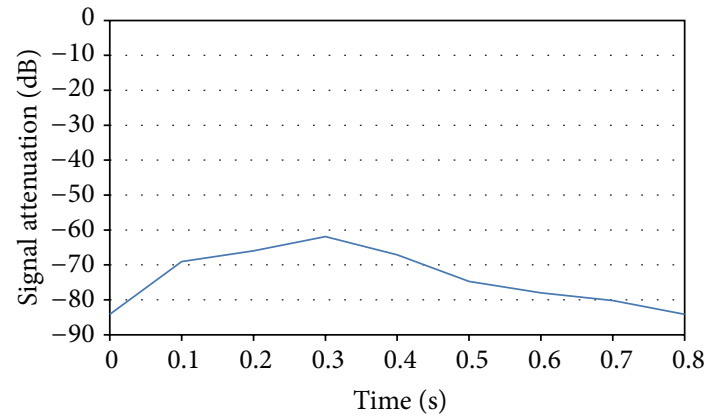

(b)

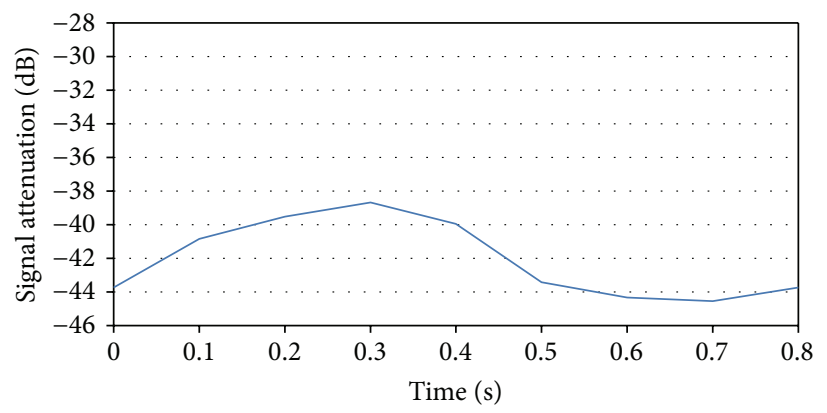

(c)

FIGURE 7: Variation of signal attenuation with time at (a) $0.1 \mathrm{GHz}$, (b) $0.5 \mathrm{GHz}$, and (c) $1 \mathrm{GHz}$, respectively.

the impedance of free space. This implies that the reflected pulses have an inverse relationship with respect to the incident pulses.

(ii) Signal Attenuation. The backward reflected power from the heart wall (interface-6), that is, the heart-lung interface $\left(P_{6 r}^{-}\right)^{\prime}$, out of the total power input at the chest surface (interface-1) $P_{i}$, is defined as the signal attenuation due to the UWB pulse echo for the frequencies in the entire band of 0.1 to $10.5 \mathrm{GHz}$ and can be determined by using (8) as given below. The attenuation factor product and the transmission coefficient product can be evaluated by using (9) and (10), respectively:

$$
\begin{aligned}
\left(P_{6 r}^{-}\right)^{\prime}= & \left|\Gamma_{6}\right|^{2} \times(\text { attenuation factor product })^{2} \\
& \times(\text { transmission coefficient product })^{2} P_{i},
\end{aligned}
$$

where

attenuation factor product

$$
=e^{-2 \alpha_{2} \ell_{2}} e^{-2 \alpha_{3} \ell_{3}} e^{-2 \alpha_{4} \ell_{4}} e^{-2 \alpha_{5} \ell_{5}} e^{-2 \alpha_{6} \ell_{6}},
$$

transmission coefficient product

$$
\begin{aligned}
= & {\left[\left(1-\left|\Gamma_{1}\right|^{2}\right)\left(1-\left|\Gamma_{2}\right|^{2}\right)\left(1-\left|\Gamma_{3}\right|^{2}\right)\right.} \\
& \left.\cdot\left(1-\left|\Gamma_{4}\right|^{2}\right)\left(1-\left|\Gamma_{5}\right|^{2}\right)\right] .
\end{aligned}
$$

The subscripts $2,3,4,5$, and 6 refer to the layers such as skin, fat, muscle, cartilage, and lung, respectively, for the calculation of the attenuation factor product and the subscripts 1, 2, 3, 4, and 5 refer to skin-fat, fat-muscle, musclecartilage, cartilage-lung, and lung-heart interfaces, respectively, for the calculation of the transmission coefficient product. Thus, the signal power attenuation is calculated for some typical frequencies in the band of 0.1 to $10.5 \mathrm{GHz}$ using (8) through (10).

The model predicted average attenuation is $-98 \mathrm{~dB}$ at $3.1 \mathrm{GHz}$ which is in good agreement with the attenuation of $-93 \mathrm{~dB}$ at this frequency as predicted by [2] but for a different thorax model. Similarly, the model predicted attenuation is $-97.8 \mathrm{~dB}$ at $3 \mathrm{GHz}$ and is in good agreement with the attenuation of $-100 \mathrm{~dB}$ at this frequency as predicted by [4].

At different instants of time in the cardiac cycle, heart assumes different dimensions. In other words, the dimensions of the layer representing heart are related to the dimension of heart. Therefore, the variation of attenuation with dimension is directly related to the variation of the heart dimensions with time. Hence, the attenuation characteristic for different dimensions is related to the heart movement. Figures $7(\mathrm{a})$ through $7(\mathrm{c})$ represent the variation of attenuation for typical frequencies of $0.1,0.5$, and $1 \mathrm{GHz}$ with time, respectively, and the time has been associated with the width of the heart (Figure 2). Thus, the period between the maximum attenuations corresponds to the heart beat period. In the case of $0.1 \mathrm{GHz}$, the minimum attenuation occurs at the heart dimension equal to $100.4 \mathrm{~mm}$ corresponding to the instant of 0.3 seconds and the maximum attenuation takes place at $111.4 \mathrm{~mm}$ at the end of diastole as depicted in Figure 7(a). The similar behavior is also observed at other 


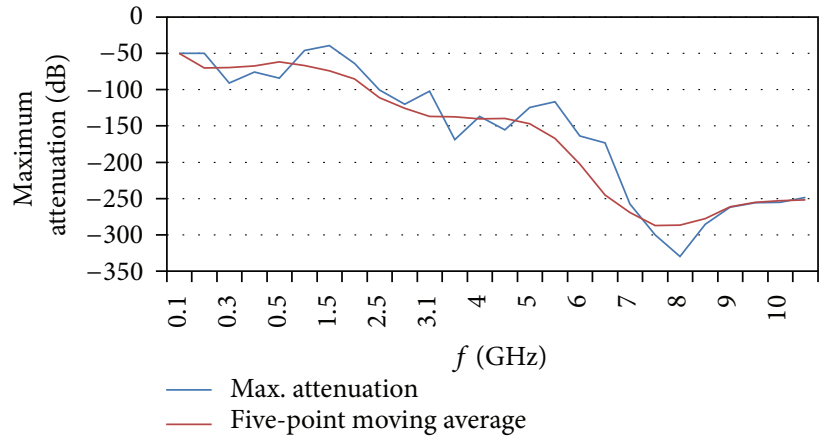

(a)

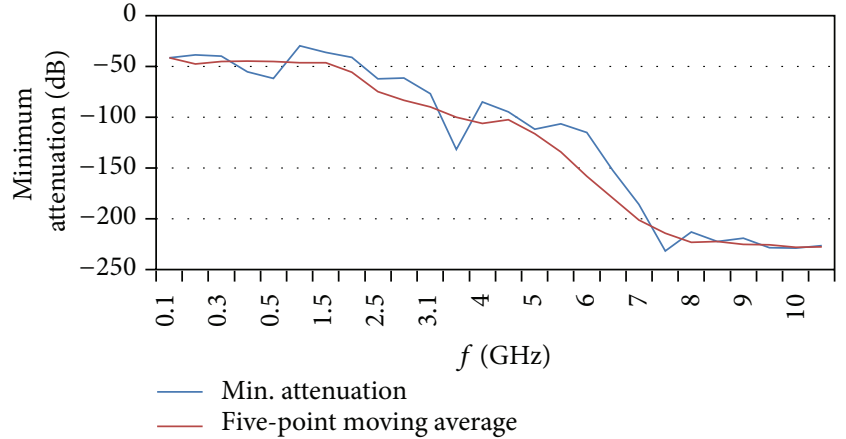

(b)

FIGURE 8: Variations of (a) maximum and (b) minimum signal attenuation with frequency. Superimposed five-point moving average is plotted.

frequencies, for example, $0.5 \mathrm{GHz}, 1 \mathrm{GHz}$, and so forth, as shown in Figures $7(\mathrm{~b})$ and $7(\mathrm{c})$, respectively.

This model predicted attenuation is obtained separately for every instantaneously changing dimension of heart during the cardiac cycle. It is found that there is a maximum and a minimum value of attenuation corresponding to every frequency in the band of 0.1 to $10.5 \mathrm{GHz}$ as shown in Figure 8 . Superimposed is the five-point moving average plot. It is observed that the points of maximum and minimum attenuation shift with frequency as depicted in Figures 8(a) and $8(\mathrm{~b})$, respectively.

From this it may be noted that there is periodic variation in attenuation of an active heart at a given frequency. The periodicity of attenuation characteristics (period between maximum attenuation and minimum attenuation) refers to the heartbeat period. Knowing the period of maximum attenuation or minimum attenuation one will be able to decide the health of the heart. Lack of periodicity might indicate the problem of an unhealthy heart. This attenuation measurement can be carried out on persons who are not accessible unlike other methods using stethoscope, electrocardiograph (ECG), and so forth.

\section{Conclusions}

Electromagnetic response of the human tissue is highly frequency dependent. Of all the body tissues encountered in the path of propagation, heart is the only moving element that can have a noticeable displacement and all others are static. Therefore, in the wake of the study of propagation characteristics, that is, signal attenuation and reflection coefficient, we focused computation of these parameters with changing dimensions of heart during a complete cardiac cycle. Then, the change of attenuation and the reflection coefficient corresponding to the change of heart size during relaxation-contraction-relaxation (one cardiac cycle) at different instants of time during the cardiac period was studied. This can provide good information about the state of a person's heart whether healthy or unhealthy. Any noticeable change of attenuation shall indicate that the person is live while no change of attenuation found in this way might lead to an unusual guess that the person might be dead.
In this paper, we have presented a one-dimensional electromagnetic model of human body and incorporated the electromagnetic properties of significant body tissues beyond heart corresponding to all frequencies from 0.1 to $10.5 \mathrm{GHz}$ to accommodate the FCC defined UWB. Moreover, the analysis is performed for changes with time of heart dimension, not for a fixed heart dimension as in earlier models. A study of variation of signal attenuation due to the instantaneous change of heart dimensions during a cardiac cycle can provide reliable information about the health of heart. This feature of change of signal attenuation may also be used to study the performance of cardiac activity of persons buried under the rubbles of the debris of a collapsed building, persons behind a wall, and so forth.

\section{Conflict of Interests}

The authors declare that there is no conflict of interests regarding the publication of this paper.

\section{References}

[1] E. M. Staderini, "UWB radars in medicine," IEEE Aerospace and Electronic Systems Magazine, vol. 17, no. 1, pp. 13-18, 2002.

[2] G. Varotto and E. M. Staderini, "On the UWB medical radars working principles," International Journal of Ultra Wideband Communications and Systems, vol. 2, no. 2, pp. 83-93, 2011.

[3] K. N. Sahu, C. D. Naidu, and K. Jaya Sankar, "Frequency dependent planar electromagnetic modeling of human body and theoretical study on attenuation for budget estimation of UWB radar," The Global Journal of Researches in Engineering, vol. 14, no. 3, pp. 35-44, 2014.

[4] M. Cavagnaro, E. Pittella, and S. Pisa, "UWB pulse propagation into human tissues," Physics in Medicine and Biology, vol. 58, no. 24, pp. 8689-8707, 2013.

[5] January 2010, http://www.sciencekids.co.nz/pictures/humanbody/heartdiagram.html.

[6] D. G. Gibson, T. A. Traill, and D. J. Brown, "Changes in left ventricular free wall thickness in patients with ischaemic heart disease," British Heart Journal, vol. 39, no. 12, pp. 1312-1318, 1977.

[7] K. Hergan, A. Schuster, M. Mair, R. Burger, and M. Töpker, "Normal cardiac diameters in cine-MRI of the heart," RöFo: Fortschritte auf dem Gebiete der Röntgenstrahlen und der Nuklearmedizin, vol. 176, no. 11, pp. 1599-1606, 2004 (German). 
[8] January 2010, http://www.stanford.edu/group/ccm_echocardio/ cgi-bin/mediawiki/index.php/Left_ventricle_size.

[9] T. A. Traill, D. G. Gibson, and D. J. Brown, "Study of left ventricular wall thickness and dimension changes using echocardiography," British Heart Journal, vol. 40, no. 2, pp. 162-169, 1978.

[10] S. Kaul, G. L. Wismer, T. J. Brady et al., "Measurement of normal left heart dimensions using optimally oriented MR images," American Journal of Roentgenology, vol. 146, no. 1, pp. 75-79, 1986.

[11] L. E. Hudsmith, S. E. Petersen, J. M. Francis, M. D. Robson, and S. Neubauer, "Normal human left and right ventricular and left atrial dimensions using steady state free precession magnetic resonance imaging," Journal of Cardiovascular Magnetic Resonance, vol. 7, no. 5, pp. 775-782, 2005.

[12] R. F. Rushmer and N. Thal, "The mechanics of ventricular contraction: a Cinefluorographic Study," Circulation, vol. 4, no. 2, pp. 219-228, 1951.

[13] C. Gabriel, "Compilation of the dielectric properties of body tissues at RF and microwave frequencies," Report AL/OETR-1996-0037, Occupational and Environmental Health Directorate, Radio Frequency Radiation Division, Brooks Air Force Base, San Antonio, Tex, USA, 1996.

[14] Institute for Applied Physics, "An Internet Resource for The Calculation of The Dielectric Properties of Body Tissues," Italian National Research Council, http://niremf.ifac.cnr.it/tissprop/.

[15] W. H. Hayt and J. A. Buck, Engineering Electromagnetics, Tata McGraw-Hill, Mumbai, India, 7th edition, 2006. 

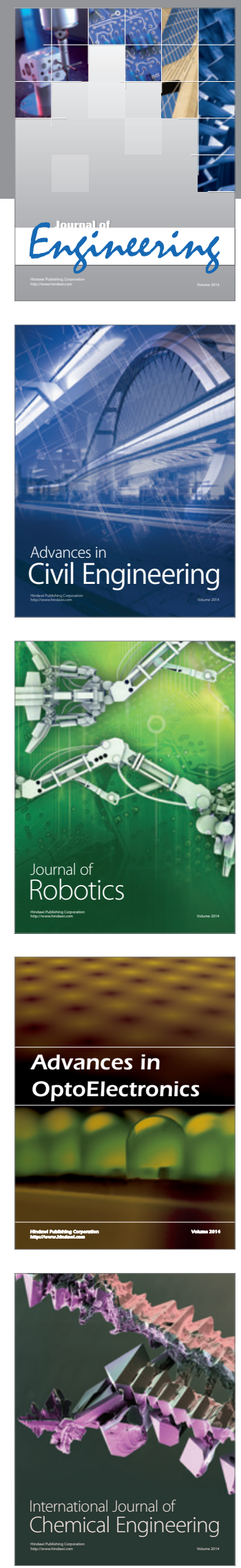

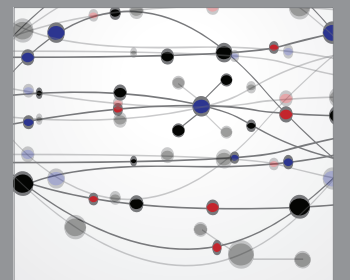

The Scientific World Journal
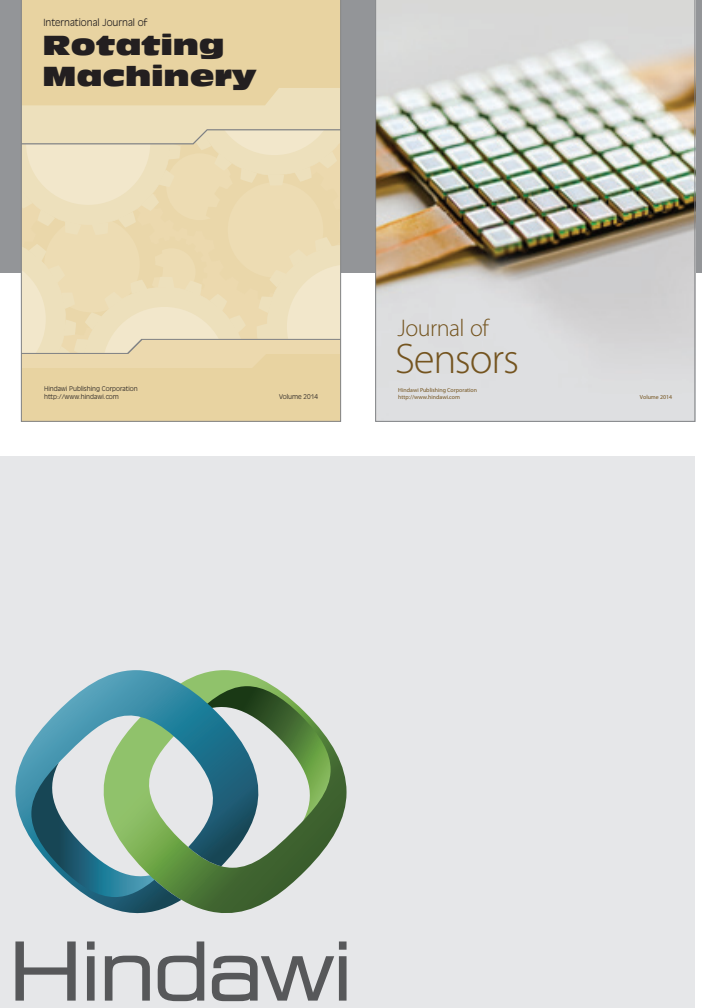

Submit your manuscripts at http://www.hindawi.com
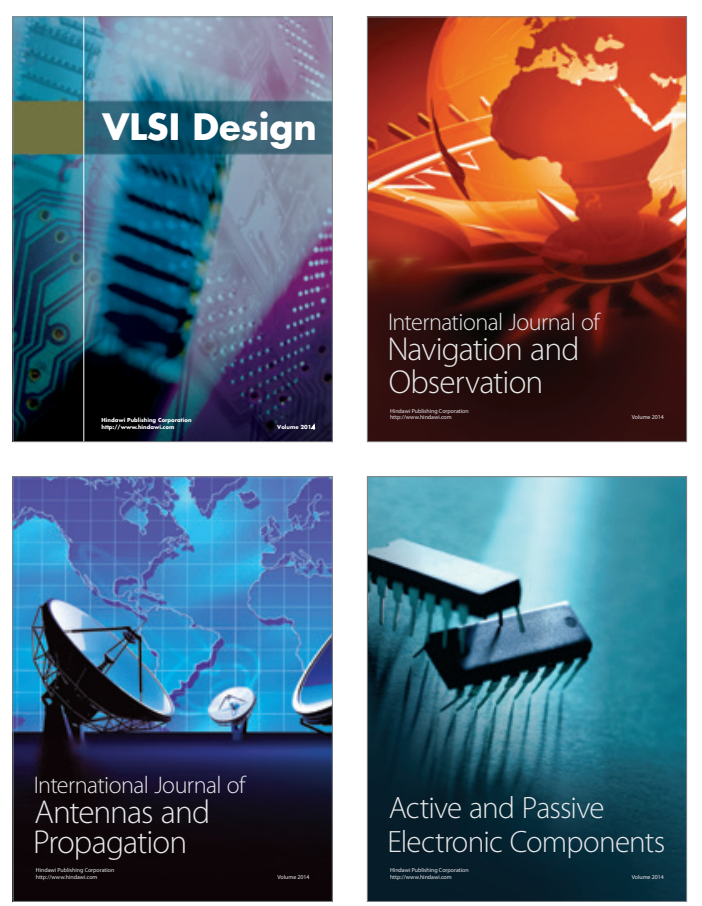
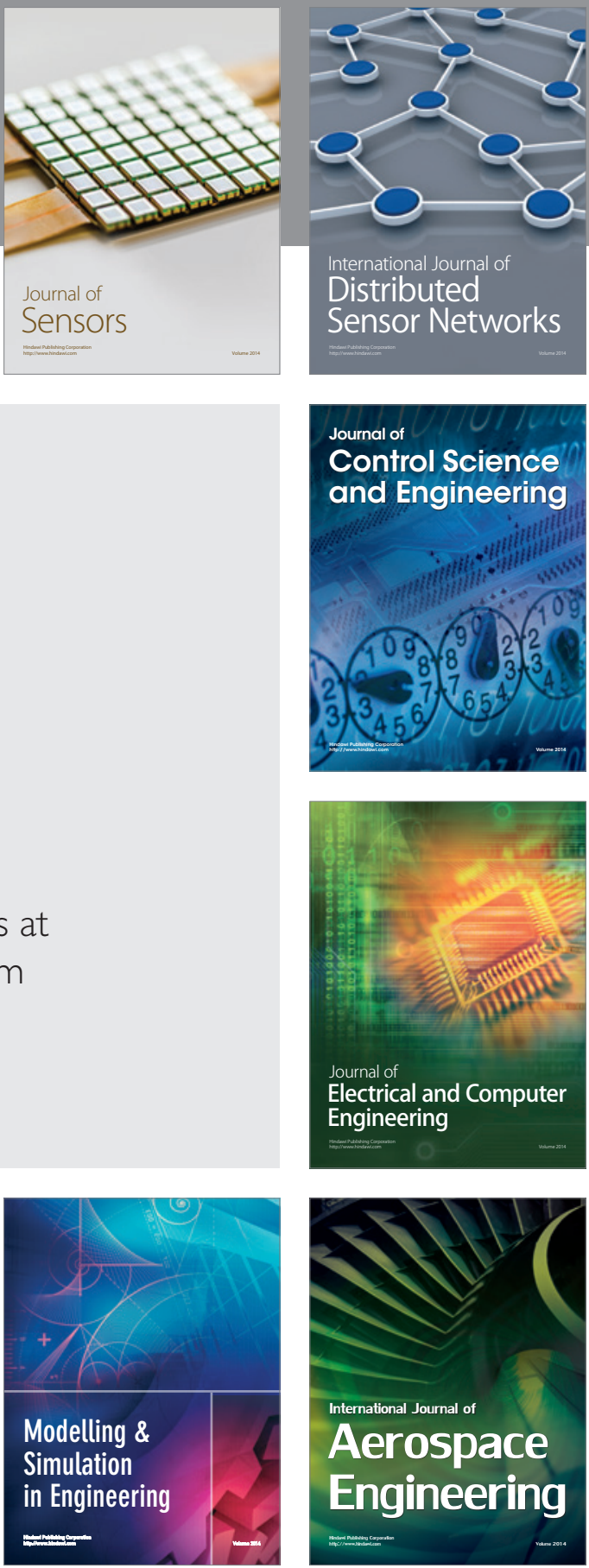

Journal of

Control Science

and Engineering
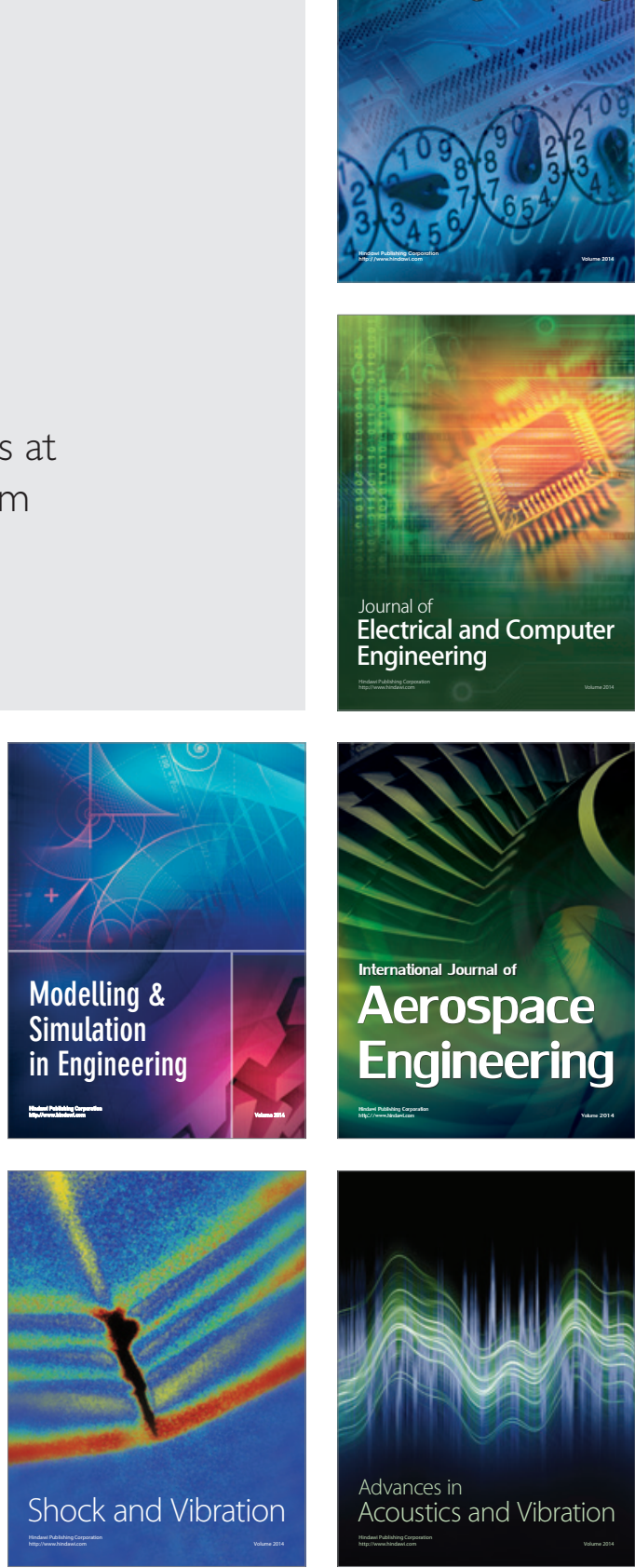